\title{
Comment on 'cosegregation of two unlinked mutant alleles in some cases of autosomal dominant familial exudative vitreoretinopathy'
}

European Journal of Human Genetics (2006) 14, 6-7. doi:10.1038/sj.ejhg.5201523; published online 30 November 2005

In a recent article in the European Journal of Human Genetics, Shastry and Trese ${ }^{1}$ reported the cosegregation of two unlinked mutant alleles in a family with autosomal dominant familial exudative vitreoretinopathy (FEVR). FEVR is inherited as a Mendelian monogenic disorder and to date five separate genes are known to underlie this condition. $^{2-6}$ Although FEVR has a penetrance of $100 \%$ there is a high degree of inter- and intra-familial phenotypic variability. As such it is not uncommon for severely affected patients to be registered blind from a young age, whereas mildly affected individuals can be completely asymptomatic. In their study, Shastry and Trese speculated that this variable phenotype may be due to oligogenic inheritance, with specific alleles at multiple loci affecting the severity and range of features seen in a patient. In particular, they hypothesised that factor $\mathrm{V}$ Leiden may be one of these modifying alleles and undertook a study to assess if it had any role in the pathogenesis of FEVR. ${ }^{1}$

Shastry and Trese screened for the presence of factor $\mathrm{V}$ Leiden in 14 unrelated FEVR families and identified it in only one. Coincidentally, this was the only family out of the 14 screened with a known mutation (c15011502 delCT) within the frizzled-4 gene (FZD4), ${ }^{2}$ mutations in which are known to account for approximately $20-40 \%$ of FEVR cases. ${ }^{2,7,8}$ Both mutations were present in all five affected members of this family but were not in three unaffected individuals, indicating that the two unlinked mutant alleles were cosegregating. Furthermore, all these mutation carrying individuals had suffered retinal detachments at an early age, suggesting that their phenotype was particularly severe. Unfortunately, the authors were not able to determine the contribution each gene made to the FEVR phenotype because all affected individuals inherited both mutations and they did not have any additional individuals with inherited mutations in only one of these genes.

However, in their paper Shastry and Trese ${ }^{9,10}$ failed to mention the widely reported observation that approximately $5 \%$ of the population are carriers of the factor $\mathrm{V}$ Leiden allele. In fact, the authors give the impression that this is not the case by reporting its exclusion in 40 control individuals. ${ }^{1}$ The family detailed in their report was of European descent ${ }^{2}$ and population studies have shown that factor $\mathrm{V}$ Leiden has highest frequency in this ethnic background, with figures suggesting that between 3 and $10 \%$ of Europeans are heterozygous carriers of the allele. ${ }^{9,11-13}$ Shastry and Trese identified the factor $\mathrm{V}$ Leiden allele in one of the 14 families they examined, giving a frequency of $7 \%(1 / 14)$ which is consistent with published figures. Furthermore, the fact that factor $\mathrm{V}$ Leiden segregates with the disease in their pedigree is not statistically significant as the family is small. Two-point linkage analysis of factor $\mathrm{V}$ Leiden with FEVR produces a maximum LOD score of only 1.49 at theta $=0$ well below 3 , the required level of significance. We therefore questioned whether the reported association of FZD4 and Leiden mutations in a single small FEVR family could be purely due to chance.

To answer this question, we screened 14 unrelated families with various forms of FEVR for the presence of factor V Leiden. We used the same method as Shastry and Trese, which identifies the mutation by the loss of an MnlI cleavage site, and confirmed detection of the allele in a panel of controls before initiating the study. Ethical approval was obtained from the Leeds Teaching Hospitals Trust Research Ethics Committee. In eight families with known mutations in $F Z D 4,{ }^{8}$ our analysis showed that none of the 64 individuals tested, 33 of whom carried FZD4 mutations, contained the Leiden allele. Included in this group were six affected members of a North American family segregating the same two-bp deletion in FZD4 as that found in the family reported by Shastry and Trese, including some with retinal detachment. ${ }^{8}$ The lack of a factor $\mathrm{V}$ Leiden allele in this family indicates that this two-bp deletion in FZD4 alone is sufficient to cause FEVR, ruling out the possibility that the reported effect of the factor V Leiden allele was specific for this mutation. The other FEVR families screened for the Leiden mutation include one family from the EVR3 locus, ${ }^{4}$ three families from the EVR4 locus, ${ }^{5,14}$ a further autosomal dominant family in whom linkage to the known loci had been 
excluded $^{6}$ and a recessive family in whom no linkage analysis had been undertaken. ${ }^{15}$

We agree with Shastry and Trese in that the variable phenotype observed in FEVR patients could well be due to modifying genes at other loci as well as environmental effects. However, to prove such a link, evidence must be presented showing that individuals containing two mutant alleles consistently have a different phenotype (either more severe or milder) than those with only one. The results presented by Shastry and Trese are interesting but anecdotal, since they provide no statistically significant evidence that factor V Leiden has an effect on the FEVR phenotype. We understand that the authors have very carefully worded their discussion so that they do not actually come to any conclusions about their finding and only suggest possibilities, but the fact that this cosegregation could be simply due to chance is not discussed. Indeed, the authors state that 'the cosegregation of unlinked genes in such a small family is statistically unlikely'. As we have shown, this statement is not supported by the data these authors presented. Furthermore, the lack of Leiden mutations in our FEVR patient cohort suggests that the factor V Leiden does not play a significant role in FEVR severity and that further studies are needed to dissect out the complexities of the variable phenotypes observed in FEVR patients.

\section{Acknowledgements}

This work was supported by The Royal Society, The Wellcome Trust and Yorkshire Eye Research.

$$
\begin{array}{r}
\text { Helen M Bottomley }{ }^{1} \text {, Louise M Downey }{ }^{1} \\
\text { Chris F Inglehearn }{ }^{1} \text { and Carmel Toomes }{ }^{*, 1} \\
{ }^{1} \text { Molecular Medicine Unit, School of Medicine, University of } \\
\text { Leeds, UK }
\end{array}
$$

${ }^{*}$ Correspondence: $\operatorname{Dr} C$ Toomes, Molecular Medicine Unit, Clinical Sciences Building, Level 6, St. James's University Hospital, Leeds, LS9 7TF, UK. Tel: +44113206 6612; Fax: +44113 2444475; E-mail: c.toomes@leeds.ac.uk

\section{References}

1 Shastry BS, Trese MT: Cosegregation of two unlinked mutant alleles in some cases of autosomal dominant familial exudative vitreoretinopathy. Eur J Hum Genet 2004; 12: 79-82.

2 Robitaille J, MacDonald MLE, Kaykas A et al: Mutant Frizzled-4 disrupts retinal angiogenesis in familial exudative vitreoretinopathy. Nat Genet 2002; 32: 326-330.

3 Chen ZY, Battinelli EM, Fielder A et al: A mutation in the Norrie disease gene (NDP) associated with X-linked familial exudative vitreoretinopathy. Nat Genet 1993; 5: 180-183.

4 Downey LM, Keen TJ, Roberts E, Mansfield DC, Bamashmus M, Inglehearn CF: A new locus for autosomal dominant familial exudative vitreoretinopathy maps to chromosome $11 \mathrm{p} 12-13$. Am J Hum Genet 2001; 68: 778-781.

5 Toomes C, Bottomley HM, Jackson RM et al: Mutations in LRP5 or FZD4 underlie the common FEVR locus on chromosome 11q. Am J Hum Genet 2004; 76: 721-730.

6 Toomes C, Downey LM, Bottomley HM, Mintz-Hittner HA, Inglehearn CF: Further evidence of genetic heterogeneity in familial exudative vitreoretinopathy; exclusion of EVR1, EVR3 and EVR4 in a large autosomal dominant pedigree. $\mathrm{Br} J$ Ophthalmol 2005; 89: 194-197.

7 Kondo H, Hayashi H, Oshima K, Tahira T, Hayashi K: Frizzled 4 gene (FZD4) mutations in patients with familial exudative vitreoretinopathy with variable expressivity. $\mathrm{Br} J$ Ophthalmol 2003; 87: 1291-1295.

8 Toomes C, Bottomley HM, Scott S et al: Spectrum and frequency of FZD4 mutations in familial exudative vitreoretinopathy (FEVR). Invest Ophthalmol Vis Sci 2004; 45: 2083-2090.

9 Rees DC, Cox M, Clegg JB: World distribution of factor V Leiden. Lancet 1995; 346: 1133-1134.

10 Ridker PM, Miletich JP, Hennekens $\mathrm{CH}$, Buring JE: Ethinic distribution of factor $\mathrm{V}$ Leiden in 4047 men and women. JAMA 1997; 277: 1305-1307.

11 Koster T, Rosendaal FR, de Ronde H, Briet E, Vandenbroucke JP, Bertina RM: Venous thrombosis due to poor anticoagulant response to activated protein C: Leiden Thrombophilia Study. Lancet 1993; 342: 1503-1506.

12 Braun A, Muller B, Rosche AA: Population study of the G1691A mutation (R506Q, FV Leiden) in the human factor $\mathrm{V}$ gene that is associated with resistance to activated protein C. Hum Genet 1996; 97: 263-264.

13 Schroder W, Koessling M, Wulff K, Wehnert M, Herrmann FH World distribution of factor V Leiden mutation. Lancet 1996; 347: $58-59$.

14 Toomes C, Downey LM, Bottomley HM et al: Identification of a fourth locus (EVR4) for familial exudative vitreoretinopathy (FEVR). Mol Vis 2004; 10: 37-42.

15 Downey LM, Sheridan E, Bradbury J, Inglehearn CF, Toomes C: Autosomal recessive inheritance of familial exudative vitreoretinopathy. Invest Ophthalmol Vis Sci 2004; 45 (Suppl): 4760.

\section{Reply to Bottomley et al}

European Journal of Human Genetics (2006) 14, 7-8. doi:10.1038/sj.ejhg.5201518; published online 16 November 2005

We welcome additional studies by Dr Bottomley et al on factor V Leiden mutation in other FEVR families and happy to note that they find our report 'interesting'. However, it is not surprising that they did not find additional families containing this mutation in their cohort. We have discussed in our short report most of the limitations of our study mentioned by Bottomley et al in their comments. Additionally, as correctly stated by Bottomley et al, we have not claimed the effect of mutation on phenotype or its association in other FEVR families but we speculated and 